\title{
Time-Varying Loads of Co-Axial Rotor Blade Crossings
}

\author{
Natasha L. Schatzman \\ NASA Ames Research Center \\ Narayanan Komerath \\ Georgia Institute of Technology \\ Ethan A. Romander \\ NASA Ames Research Center
}

\begin{abstract}
The blade crossing event of a coaxial counter-rotating rotor is a potential source of noise and impulsive blade loads. Blade crossings occur many times during each rotor revolution. In previous research by the authors, this phenomenon was analyzed by simulating two airfoils passing each other at specified speeds and vertical separation distances, using the compressible Navier-Stokes solver OVERFLOW. The simulations explored mutual aerodynamic interactions associated with thickness, circulation, and compressibility effects. Results revealed the complex nature of the aerodynamic impulses generated by upper/lower airfoil interactions. In this paper, the coaxial rotor system is simulated using two trains of airfoils, vertically offset, and traveling in opposite directions. The simulation represents multiple blade crossings in a rotor revolution by specifying horizontal distances between each airfoil in the train based on the circumferential distance between blade tips. The shed vorticity from prior crossing events will affect each pair of upper/lower airfoils. The aerodynamic loads on the airfoil and flow field characteristics are computed before, at, and after each airfoil crossing. Results from the multiple-airfoil simulation show noticeable changes in the airfoil aerodynamics by introducing additional fluctuation in the aerodynamic time history.
\end{abstract}

CITATION: Schatzman, N., Komerath, N., and Romander, E., "Time-Varying Loads of Co-Axial Rotor Blade Crossings," SAE Int. J. Aerosp. 10(2):2017, doi:10.4271/2017-01-2024.

\section{INTRODUCTION}

Coaxial rotor aircraft are gaining interest in civil and military applications, as well as in the small, Unmanned Aerial Vehicle (UAV) market. Compared to single-main rotor or tiltrotor configurations, however, there are fewer studies, analytical or experimental, on coaxial rotors. Studies on coaxial rotor performance through 1997 are summarized in Coleman [1]. Barbely et al. [2] provides a compilation of computational studies of coaxial rotors in hover and forward flight; references for the data used to validate the current studies are also cited.

The approach to explore a coaxial rotor in 2D, developed by Barbely et al. $[\underline{2}, \underline{3}$, and $\underline{4}]$, was pursued in a recent paper by Singh and Friedmann [5]. Using vortex discretized airfoils, Singh and Friedmann's 2D simulations included effects of downwash and shed vorticity by using periodic boundaries. The loads on the upper airfoil were found to be larger compared to the lower airfoil when the airfoils crossed one another. Studies included effects of pitch angle and separation distance, where increasing the pitch angle and decreasing the vertical separation distance increased the change in lift at time of crossing. Furthermore, a 3D simulation using the rational function approximation unsteady aerodynamic loads model combined with the viscous vortex particle method was validated and results revealed a periodic vibration corresponding to the azimuthal location of blade overlap.

In a series of studies, Barbely et al. $[\underline{2}, \underline{3}$, and $\underline{4}]$ used the $2-\mathrm{D}$ version of the OVERFLOW Navier-Stokes solver to model the crossing of two airfoils, offset vertically and traveling in opposite directions. Vertical spacing between the airfoils, airfoil angle of attack, Mach number (including transonic and compressible cases), and airfoil thickness were varied. The results showed dramatic effects on the aerodynamic loads on the two airfoils, as well as effects on the pressure field surrounding the airfoils during and after the crossing. In this previous study, effects of circulation, thickness, and compressibility were retained, while the effects of shed vorticity and downwash were not modeled. Results revealed that circulation was the dominating aerodynamic source compared to thickness effects. Effects of compressibility are only important for high Mach numbers, which most modern rotors are designed to avoid.

The present effort continues the work of Barbely et al. [4], expanding the complexity of the 2D simulation by replacing the two-airfoil scenario with a "train" of airfoils, vertically offset, traveling in opposite 
directions. The train of airfoils is more representative of a coaxial rotor system with each rotor having multiple blades, thus producing numerous blade crossing events in one rotor revolution. The goal of this study is to understand the effect of the shed vorticity of multiple airfoils on the surrounding flow field and on the airfoil loading.

A description of the OVERFLOW analysis, including the gridding approach, is presented first. The Results section includes key findings from the two-airfoil crossing simulation presented in Barbely et al. [4]. A more detailed analysis of the airfoil shed wake is also presented. The two-airfoil results provided the motivation for exploring the train of airfoils (eight-airfoils in each train). Two different coaxial rotor geometries are simulated using the 2D analogy. Loading on an airfoil before, during, and after a crossing event is compared for a two-airfoil system and a system with a train of airfoils. Results for an isolated airfoil are included as a baseline. Finally, specific observations are presented. A graphical description (not to scale) of the three simulation set ups are seen in Fig. 1. The isolated airfoil simulation (Fig. $1 \mathrm{a})$ ) is denoted by "ISO", the two airfoil simulation (ig. 1 b) ) airfoils are denoted by "UA" for the upper airfoil and "LA" for the lower airfoil, and the multi airfoil simulation (Fig. $1 \mathrm{c})$ ) is denoted by "UA1" through "UA8" for the upper airfoils and "LA1" through "LA8" for the lower airfoils. The abbreviations for each set up and airfoil are used throughout the paper.

ISO

a.

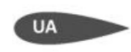

LA

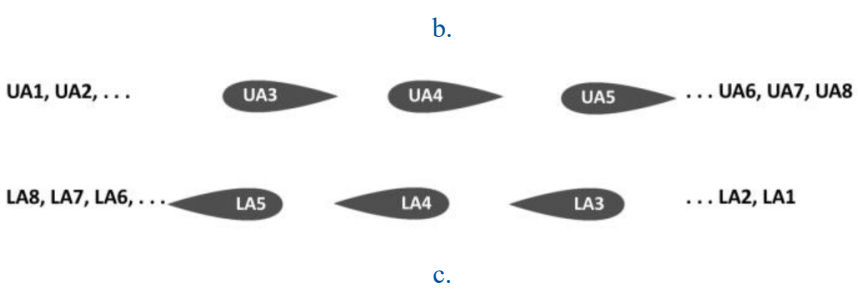

Figure 1. Pictorial representation of a) isolated, b) two airfoils crossing, and c) multi airfoils crossing simulations.

\section{ANALYSIS DESCRIPTION}

Fluctuations in the aerodynamic loading during a blade crossing event result in vorticity shed into the airfoil wake. This vorticity rolls up and persists in the flowfield long enough for it to interact with trailing blades/airfoils. The present work modified the prior model to track these shed vortices and examine their interaction with trailing blades/airfoils.

OVERFLOW 2.2k []], developed by NASA, is a compressible Reynolds-Averaged Navier-Stokes CFD analysis tool that uses structured, overset grids. This study used OVERFLOW's 2D configuration to model airfoils moving through a static background mesh. Over the course of the simulation, these airfoil(s) converged horizontally toward the origin at a speed representative of the tip speed of the coaxial rotor design. At the origin they passed by each other to model a blade passage or "overlap". The upper airfoil(s) were initially displaced 200 chord lengths to the right of the origin while the lower airfoils were initially displaced 200 chord lengths to the left of the origin. This distance was chosen to give the flow sufficient time to reach a steady state before the airfoil grids reached the origin. The airfoils were also displaced from the origin in the vertical axis by a distance that represented the rotor-rotor separation for the given coaxial design. This vertical separation was held constant for the entire simulation.

OVERFLOW 2.2k offers a wide variety of numerical schemes, turbulence models, and boundary conditions. All simulations in this study used a $5^{\text {th }}$ order accurate central difference spatial scheme with an ARC3D diagonalized Beam-Warming scalar pentadiagonal scheme for the left hand side. Time marching was performed using a $2^{\text {nd }}$ order dual time stepping scheme. Turbulence was modeled using the Spalart-Allmaras one equation turbulence model. Airfoil surfaces were modeled as viscous, adiabatic walls, and the edges of the computational domain were modeled using a characteristic condition that imposed a quiescent freestream at standard atmospheric conditions. A physical time step was chosen such that the airfoils moved $1 / 200^{\text {th }}$ of a chord length for each time step. Each physical time step included 10 dual-time subiterations. These values ensured that subiteration convergence met or exceeded two orders of decrease in the residual at all times.

Airfoils were modeled using a set of identical body fitted, curvilinear structured grids. These grids were of an O-topology with 253 points around each airfoil and 65 points normal to the airfoil surface. The $\mathrm{y}^{+}$ value at the first point off the airfoil surface was less than one. These airfoil grids moved through a Cartesian background mesh that extended 1200 chords from the origin in the horizontal and vertical direction. The initial spacing of the background grids in the immediate vicinity of the airfoil grids was 0.02 chords in both the horizontal and vertical direction. Background grids could be refined based on an estimate of the solution error using overlapping Cartesian refinement grids (Fig. 2). This grid adaption scheme ensured sufficient grid support for shed vorticity in the wake of the airfoils. All grids were modeled in 2D. Total grid sizes ranged from approximately 350,000 points for an isolated airfoil before grid adaption to approximately 11 million for an 8 airfoil simulation after grid adaption.

Forces and moments exerted on the airfoils are integrated from the pressure and viscous stress at the airfoil surface. These quantities are integrated and recorded periodically over the entire course of the simulation. The force in the vertical, $z$, direction is positive up and is equivalent to aerodynamic lift (see Fig. 2). The coefficient of z-force is abbreviated as $c_{z}$. The direction of force in the horizontal, $x$, direction is positive toward the airfoil's trailing edge and is equivalent to aerodynamic drag. The coefficient of $\mathrm{x}$-force is abbreviated by $\mathrm{c}_{\mathrm{x}}$. 


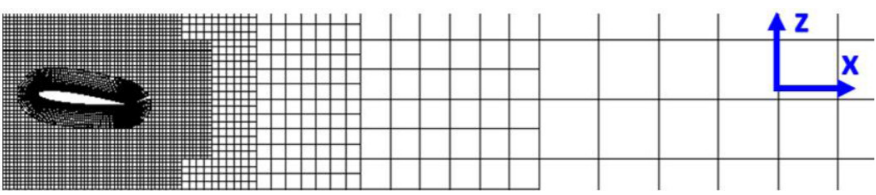

a.

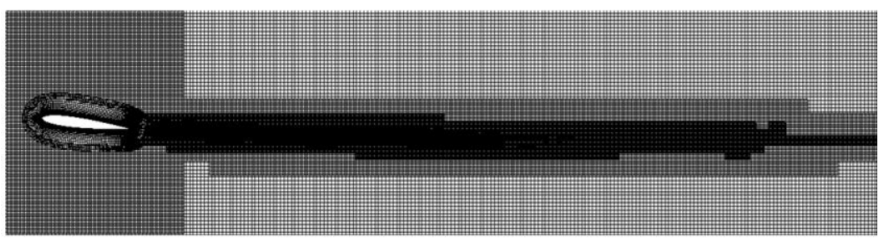

b.

Figure 2. OVERFLOW simulation a) without, and b) with grid adaption.

\section{RESULTS}

Previous calculations are revisited and recalculated to determine the importance of shed vorticity using an upgraded grid. The conclusion of this effort lead to simulating a train of airfoils to understand the effect of shed vorticity. Results from the isolated, two airfoil, and multiple airfoils crossing simulations are analyzed and compared.

\section{Simulation of two airfoils crossing}

Using the Harrington Coaxial Rotor 1 (HC1) hover conditions [7], Barbely et al. [4] performed 2D analog simulations of two airfoils crossing. Mach number, separation distance, angle of attack, and airfoil thickness were also varied in the $2 \mathrm{D}$ simulations. Note that varying Mach number is equivalent to simulating the crossing event at different radial locations, for the same rotor RPM. The time of overlap occurs when the $1 / 4$-chord location of each airfoil coincides, and is denoted by the grey line "Overlap" in presented figures. The $c_{z}$ is the force in the vertical direction at $1 / 4$ chord, while $c_{x}$ is the force in the horizontal direction at $1 / 4$ chord. The upper airfoil is denoted as UA and the lower airfoil as LA.

The 2D simulations aided in understanding the behavior of the flow field in terms of circulation (Fig 3 a $)$ ), thickness (Fig. 3 b)), and compressibility (Fig. 3 c)) effects for a coaxial rotor. Each of the three aerodynamic effects are highlighted by comparing the parameter that dominates the effect.

The effect of circulation was explored by comparing results for two angles of attack with the same symmetrical airfoil, low speed, and separation distance in order to eliminate any large effects due to thickness or compressibility. Figure 3 a) shows $c_{z}$ of two NACA 0012 airfoils crossing over time traveling at $\mathrm{M}=0.47$ and vertically separated by $2.33 \mathrm{ft}(\mathrm{S} / \mathrm{c}=6.21)$ while comparing the difference in angle of attack of $7^{\circ}$ and $0^{\circ}$. To easily compare results, the data is vertically shifted up by a $c_{z}$ of 0.85 for $\alpha=0^{\circ}$. Compared to the $0^{\circ}$ angle of attack case, an angle of attack of $7^{\circ}$ resulted in a greater change in $\mathrm{c}_{\mathrm{z}}$ before and after overlap due to circulation.

Thickness effects are explored by two different airfoils at a constant low speed, separation distance, and angle of attack, which eliminated large effects of circulation and compressibility. Figure $3 \mathrm{~b}$ ) shows $\mathrm{c}_{\mathrm{z}}$ of two airfoils crossing over time traveling at $\mathrm{M}=0.47, \alpha=0^{\circ}$, and a separation distance of $0.5 \mathrm{ft}(\mathrm{S} / \mathrm{c}=1.33)$, while comparing NACA 0012 and NACA 0001 airfoils. The two symmetric airfoils at time of crossing have an opposite and equal effect on $\mathrm{c}_{\mathrm{z}}$ and $\mathrm{c}_{\mathrm{x}}$ (not shown). This effect is caused by the finite thickness of the airfoil (thickness effects). An S/c of 1.335 is an extreme case where modern day coaxial rotors do not operate. For an S/c greater than 2, the effect due to thickness becomes insignificant; therefore thickness effects are not as significant compared to circulation effects for coaxial rotors.

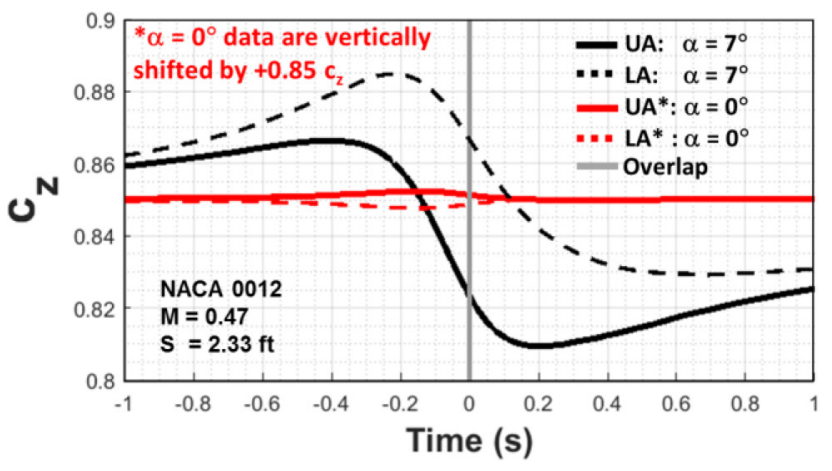

a.

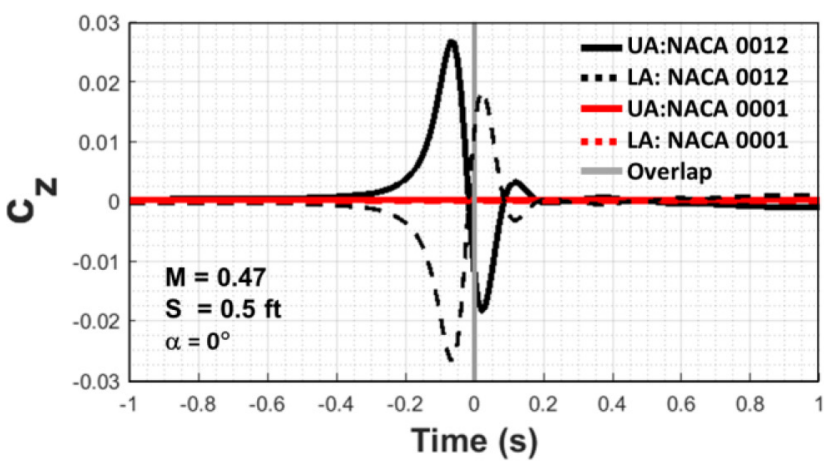

b.

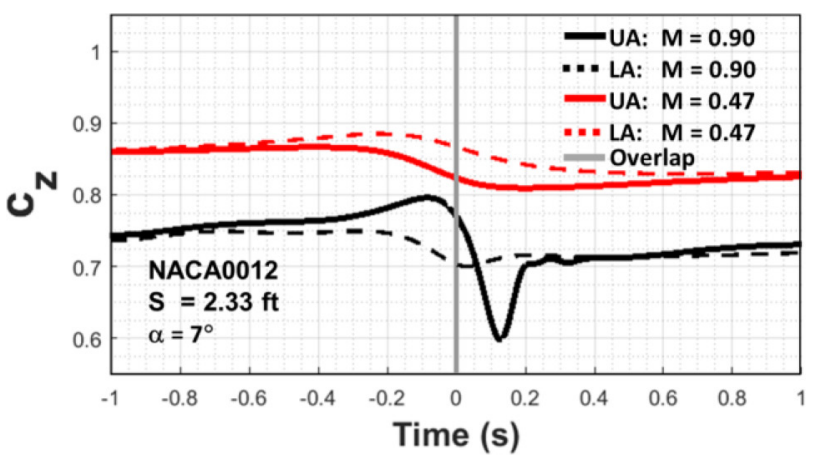

c.

Figure 3. Two airfoils crossing simulation of $\mathrm{c}_{\mathrm{z}}$ versus time to show effect of a) circulation, b) thickness, and c) compressibility.

Effects of compressibility are explored by comparing two different (incompressible and compressible) speeds with the same symmetrical airfoil, angle of attack, and separation distance to eliminate any large effects of circulation or thickness. Figure $3 \mathrm{c}$ ) shows the $\mathrm{c}_{\mathrm{z}}$ of two NACA 0012 airfoils crossing over time traveling at an angle of attack for $7^{\circ}$, separated by $2.33 \mathrm{ft}(\mathrm{S} / \mathrm{c}=6.21)$, while comparing Mach 
numbers of 0.47 and 0.90 . For large Mach numbers, at the time of overlap, the upper surface shock of the lower airfoil resulted in a larger change in $\mathrm{c}_{\mathrm{z}}$ of the upper airfoil - this result is due to compressibility effects. A large change in $\mathrm{c}_{\mathrm{z}}$ is observed at $\mathrm{M}=0.90$ compared to $\mathrm{M}=0.47$ in Fig. $3 \mathrm{c}$ ), though if large Mach numbers can be avoided, the effect of compressibility becomes insignificant.

In conclusion, in terms of circulation, thickness, and compressibility effects, the prominent aerodynamic source at low speeds is circulation. The lift of both the upper and lower airfoil, when compressibility is not dominating, increased before overlap, followed by a decrease in lift after overlap. Before overlap, the upper airfoil sees an increase in angle of attack due to the up-wash from the lower airfoil. The angle of attack of the lower airfoil also increases due to the up-wash from the upper airfoil and therefore an increase in lift is experienced by both airfoils as depicted in Fig 4 a). The opposite occurs after time of overlap, where a decrease in lift is seen for both the upper and lower airfoil (see Fig. 4 b)). As the airfoils approach one another, $c_{z}$ of each airfoil changes due to the circulation effect of the other airfoil.

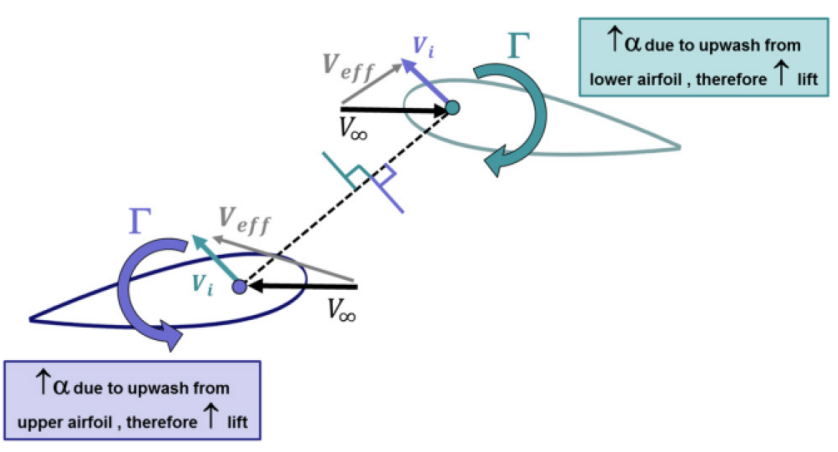

a.

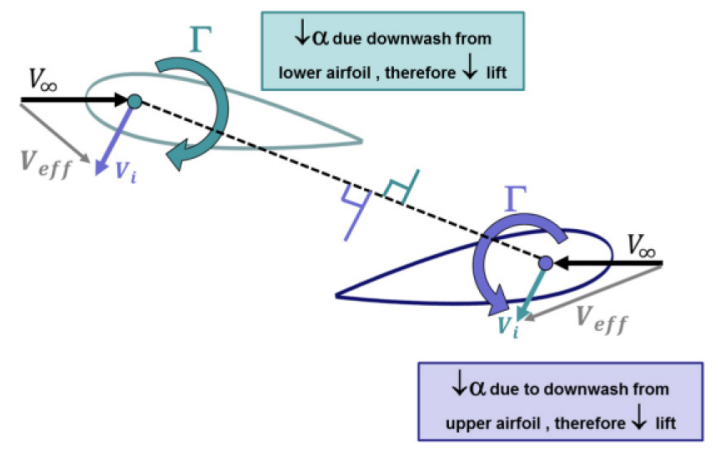

b.

Figure 4. Change in lift of two NACA0012 (when compressibility is not dominating) airfoils crossing: a) before and b) after.

Rapid changes in airfoil lift and drag, whether due to circulation, thickness and/or compressibility, result in strong shed vorticity deposited into the fluid medium. Any ensuing airfoils that impinge on these shed vortices are susceptible to additional airload fluctuations. To study this phenomenon, the two airfoils crossing in the $\alpha=7^{\circ}$ case of Fig. 3 a) was recalculated to directly capture the shed vorticity field. Grid adaption is used within OVERFLOW to preserve the shed vorticity for a longer period of time.
Figure $5 \mathrm{a}$ ) shows the recalculated $\mathrm{c}_{\mathrm{z}}$ results for the isolated and upper airfoil $\left(\mathrm{HC} 1: \mathrm{M}_{\infty}=0.47\left(\mathrm{~V}_{\text {tip }}=500 \mathrm{ft} / \mathrm{s}\right), \alpha=7^{\circ}\right.$, and $\mathrm{S} / \mathrm{c}=6.21(\mathrm{~S}=$ $2.33 \mathrm{ft}, \mathrm{c}=0.375 \mathrm{ft})$ ). The horizontal axis is in terms of chord distance to overlap; negative and positive values correspond to before and after airfoil overlap, respectively. The vertical grey line is when the $1 / 4$ chord of each airfoil overlap. The $c_{z}$ results are converted to circulation $(\Gamma)(\underline{\text { Fig. } 5 \text { b }})$ ), then the negative time derivative of circulation is computed to obtain shed vorticity (-d $/$ dt) (Fig. $5 \mathrm{c})$ ). The circulation is negated to satisfy Kelvin's circulation theorem (conservation of body forces), and the derivative of circulation is taken with respect to time (distance) due to the time varying loads. There is an increase in shed vorticity beginning about 10 chords before overlap, peaking just before overlap, and then decreasing back to zero approximately 10 chords after overlap.
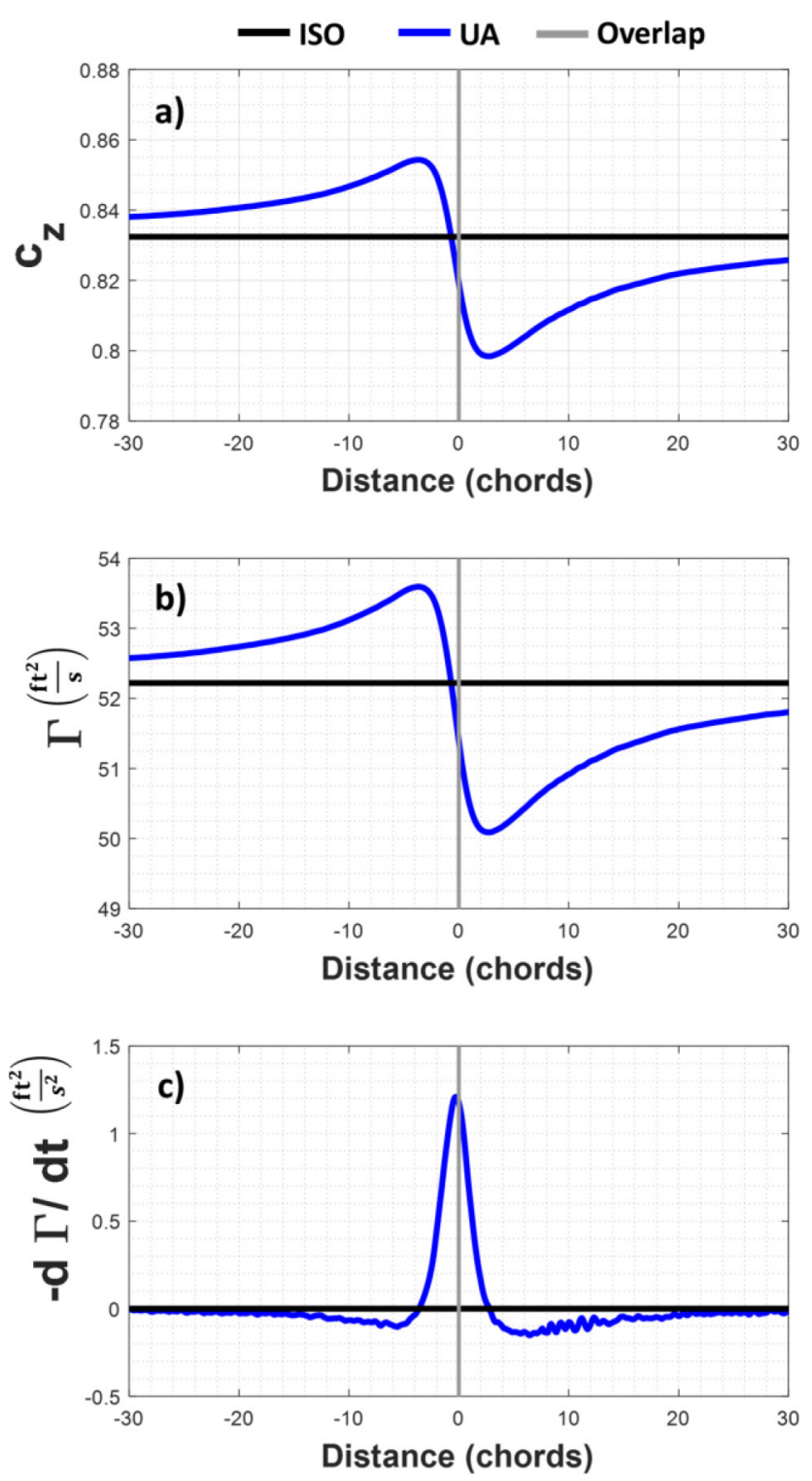

Figure 5. Isolated and two-airfoil simulation results for a) $\left.c_{z}, b\right) \Gamma$, and c) $-d \Gamma /$ $\mathrm{dt}$ versus distance to overlap for upper airfoil ( $\mathrm{HC} 1: \mathrm{M}=0.47\left(\mathrm{~V}_{\text {tip }}=500 \mathrm{ft} / \mathrm{s}\right)$, $\alpha=7^{\circ}$, and $\left.\mathrm{S} / \mathrm{c}=6.21(\mathrm{~S}=2.33 \mathrm{ft}, \mathrm{c}=0.375 \mathrm{ft})\right)$. 
The flow field solution for the isolated airfoil is subtracted from the two-airfoil system solution. This processing highlights the aerodynamics unique to coaxial rotors by removing the velocity deficit in the airfoil wake but also amplifies noise in the solution.

Figure 6 shows the difference in y-vorticity between the two-airfoil simulation (upper airfoil) and isolated airfoil simulation for a) 10 chords before overlap, b) 5 chords before overlap, c) at overlap, d) 5 chords after overlap, and e) 10 chords after overlap. The horizontal axis is the distance from the trailing edge of the airfoil, while the vertical axis is the $\mathrm{z}$-distance. The units for both axes are in chords. The vertical grey line in Fig. $6 \mathrm{~d}$ ) and e) is at the location when the $1 / 4$ chord of each airfoil overlapped. A sign change in $\Delta y$-vorticity is seen in the airfoil's wake when comparing before and at overlap. Before and at overlap in Fig 6 a), b), and c), the center of the wake is blue (positive $\Delta y$-vorticity) after overlap in Fig. $6 \mathrm{~d}$ ), and e) the center of the wake is yellow (negative $\Delta y$-vorticity). The change from positive shed vorticity (-d $\Gamma / \mathrm{dt})$ to negative follows the trend seen in Fig. $5 \mathrm{c}$ ). The change in sign change in shed vorticity is due to circulation effects from crossing.

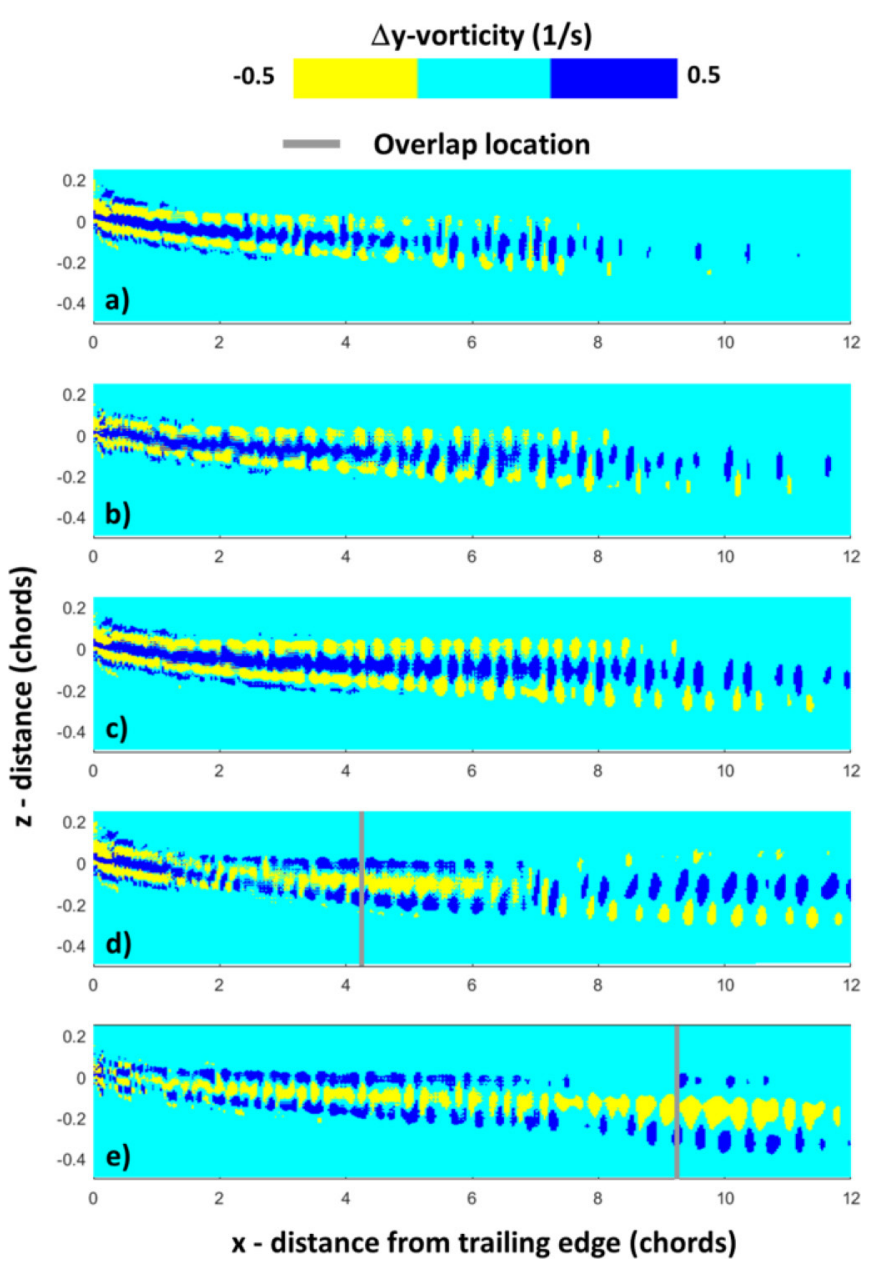

Figure 6. Difference in y-vorticity (two-airfoil simulation (UA) minus isolated airfoil (ISO) simulation): a) 10 chords before overlap, b) 5 chords before overlap, c) at overlap, d) 5 chords after overlap, and e) 10 chords after overlap. $\mathrm{HC} 1: \mathrm{M}=0.47, \alpha=7^{\circ}$, and $\mathrm{S} / \mathrm{c}=6.21(\mathrm{~S}=2.33 \mathrm{ft})$.

\section{SIMULATION OF EIGHT-AIRFOILS CROSSING}

The two airfoils traveling in opposite directions demonstrated the effects of circulation, thickness, and compressibility for a coaxial rotor but lacked any treatment of the rotor wake, due to multiple blades, unlike Singh and Friedmann who modeled downwash and shed vorticity []. Since the downwash is not currently modeled, the simulation is a more extreme case in that the shed vorticity from each airfoil remains in the plane of the rotor and is not pushed down. The investigation of shed vorticity of the two-airfoil crossing simulation (Fig. 5 and Fig. 6), therefore, provided motivation to pursue studying a coaxial rotor wake produced by a train of airfoils. Figure 7 shows a train of eight airfoils representing the upper rotor blades and eight airfoils representing the lower rotor blades. Airfoils are labeled by vertical position (upper or lower) and horizontal position. For example, upper airfoil 4 and lower airfoil 4 are denoted as UA4 and LA4. Figure 7 shows a pictorial image of a multiple airfoil simulation at time of overlap when a) UA1 and LA1, b) UA2 and LA2, and c) UA4 and LA4 are overlapped (the $1 / 4$ chord location of each airfoil coincide).

The arrangement of airfoils shown in Fig. 7 was chosen for illustrative purposes. The modeled geometry used a vertical separation between the upper and lower airfoil train equal to the rotor-rotor separation for the modeled coaxial rotor. The distance between airfoils in a train (e.g. UA3 and UA4) was set equal to the circumferential distance between the tips of the modeled rotor. A train of 8 airfoils was chosen to ensure that there was sufficient aerodynamic influence from airfoils preceding and following the airfoil of interest (UA4).

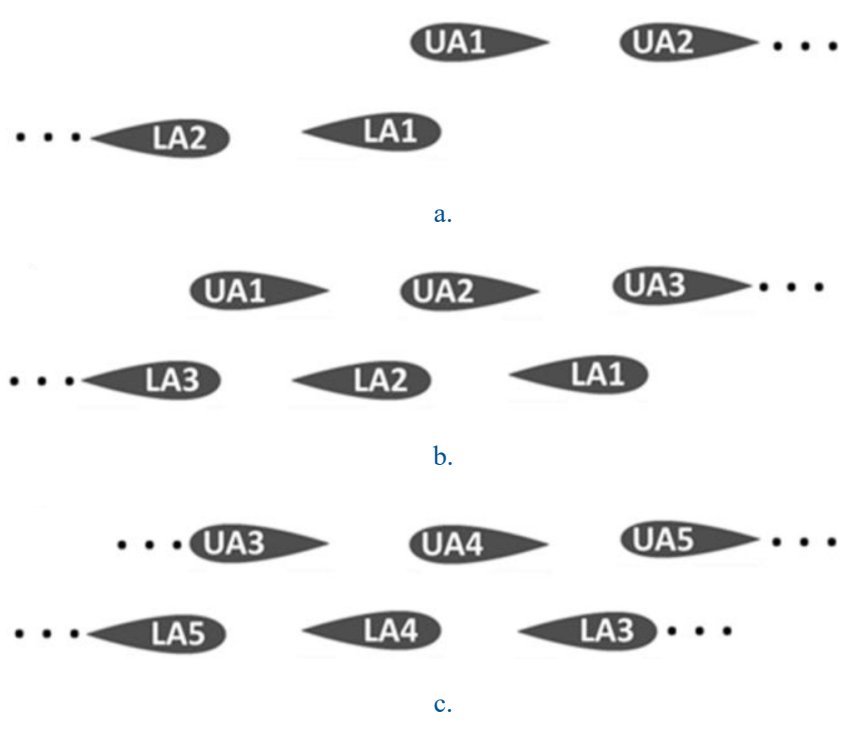

Figure 7. Multiple airfoil simulation illustration at time of overlap of a) UA1 and LA1, b) UA2 and LA2, and c) UA4 and LA4.

\section{Test Conditions for 8-Airfoil Simulation}

To investigate the effect of shed vorticity, two different rotor configurations are simulated, shown in Table 1. The Harrington coaxial rotor $1(\mathrm{HC} 1)$ was previously modeled by Barbely et al. $[\underline{1}, \underline{2}$, and 4]; HC1 was tested in a wind tunnel and the results have been used by many as a validation case [7]. The second coaxial rotor 
geometry is representative of modern coaxial systems []]. The modern coaxial rotor design has 3 blades per rotor, a smaller separation distance, and faster tip speed compared to the $\mathrm{HC} 1$.

Table 1. Simulated rotor design parameters 3D and 2D analog.

\begin{tabular}{|c|c|c|c|c|c|}
\hline \multirow{2}{*}{$\begin{array}{c}2 \mathrm{D} \\
\text { simulation }\end{array}$} & \multicolumn{2}{|c|}{ 2D simulation } & \multirow{2}{*}{$\begin{array}{c}\text { Coaxial } \\
\text { rotor } \\
\text { parameter }\end{array}$} & \multicolumn{2}{|c|}{ Coaxial rotor design } \\
\hline & $\mathrm{HC1}$ & Modern & & $\mathrm{HC1}$ & Modern \\
\hline $\begin{array}{l}\text { Radius } \\
\text { (ft) }\end{array}$ & $\mathrm{N} / \mathrm{A}$ & $\mathrm{N} / \mathrm{A}$ & $\begin{array}{l}\text { Radius } \\
\text { (ft) }\end{array}$ & 12.5 & 20 \\
\hline $\begin{array}{l}\text { No. of } \\
\text { airfoils } \\
\text { (per train) }\end{array}$ & 8 & 8 & $\begin{array}{l}\text { No. of } \\
\text { blades } \\
\text { (per rotor) }\end{array}$ & 2 & 3 \\
\hline $\begin{array}{l}\text { S, vertical } \\
\text { separation } \\
\text { between } \\
\text { airfoils } \\
\text { (ft) }\end{array}$ & 2.33 & 2 & $\begin{array}{l}\mathrm{S} \text {, rotor } \\
\text { separation } \\
(\mathrm{ft})\end{array}$ & 2.33 & 2 \\
\hline $\begin{array}{l}\mathrm{D}, \\
\text { horizontal } \\
\text { separation } \\
\text { between } \\
\text { airfoils } \\
\text { (ft) } \\
\end{array}$ & 39.27 & 41.9 & $\begin{array}{l}\mathrm{D}, \\
\text { horizontal } \\
\text { separation } \\
\text { (ft) }\end{array}$ & N/A & N/A \\
\hline $\begin{array}{l}\mathrm{c}, \text { chord } \\
\text { (ft) }\end{array}$ & 0.375 & 0.5 & $\begin{array}{l}\text { c, chord } \\
\text { (ft) }\end{array}$ & varying & 0.5 \\
\hline $\begin{array}{l}\text { Airfoil } \\
\text { speed } \\
(\mathrm{ft} / \mathrm{s})\end{array}$ & 500 & 700 & $\begin{array}{l}\mathrm{V}_{\text {tip }} \text {, hover } \\
(\mathrm{ft} / \mathrm{s})\end{array}$ & 500 & 700 \\
\hline $\begin{array}{l}\text { M, Mach } \\
\text { number }\end{array}$ & 0.47 & 0.627 & $\begin{array}{l}\text { M, Mach } \\
\text { number } \\
\text { (M }_{\text {tip }} \text { for }\end{array}$ & 0.47 & 0.627 \\
\hline $\begin{array}{l}\text { Linear } \\
\text { twist } \\
\text { (deg) } \\
\end{array}$ & $\mathrm{N} / \mathrm{A}$ & $\mathrm{N} / \mathrm{A}$ & $\begin{array}{l}\text { Linear } \\
\text { twist } \\
\text { (deg) }\end{array}$ & 0 & -8 \\
\hline$\alpha(\mathrm{deg})$ & 7 & 5 & $\alpha(\operatorname{deg})$ & varying & N/A \\
\hline$\theta_{0}(\operatorname{deg})$ & N/A & N/A & $\theta_{0}(\operatorname{deg})$ & 7 & 0 \\
\hline Airfoils & $\begin{array}{c}\text { NACA0 } \\
012\end{array}$ & $\begin{array}{c}\text { NACA } \\
0012\end{array}$ & Airfoils & varying & varying \\
\hline
\end{tabular}

\section{COMPARISON BETWEEN 2- AND 8-AIRFOIL SIMULATIONS}

The changes in $c_{z}$ and $c_{x}$ are analyzed over time with respect to distance in chords to overlap. An isolated airfoil, two airfoils crossing, and eight airfoils crossing are compared to understand how the aerodynamics change for each overlap occurrence. The simulation case with two airfoils passing each other is provided for comparison in subsequent plots and denoted as "2 Airfoils" - see Fig. 9, for example.
The 2D OVERFLOW simulations for the Harrington coaxial rotor 1 and the modern coaxial design are analyzed by comparing $\mathrm{c}_{\mathrm{z}}$ and $\mathrm{c}_{\mathrm{x}}$ versus distance (in chords) to overlap for each upper and lower airfoil. The negative and positive distances represent before and after time of overlap, respectively.

\section{Simulation of Harrington Rotor 1}

The Harrington rotor 1 is simulated using eight NACA0012 upper and lower airfoils at $\mathrm{M}=0.47\left(\mathrm{~V}_{\text {tip }}=500 \mathrm{ft} / \mathrm{s}\right), \alpha=7^{\circ}, \mathrm{S} / \mathrm{c}=6.21(\mathrm{~S}=$ $2.33 \mathrm{ft}, \mathrm{c}=0.375 \mathrm{ft})$, and $\mathrm{D} / \mathrm{c}=104.72(\mathrm{D}=39.27 \mathrm{ft})$. The $\mathrm{c}_{\mathrm{z}}$ time history for each upper airfoil in the multi airfoil simulation is analyzed at the overlap occurrence between UA4 and LA4. Figure 8 shows $\mathrm{c}_{\mathrm{z}}$ versus distance to overlap for UA4 in the multi airfoil simulation of $\mathrm{HC} 1$, overlap of UA4 and LA4 occur at a distance of 0 chords along the $\mathrm{x}$-axis. In Fig. 8, three large peaks are shown before the first overlap this is because UA4 passes through the starting vortex of UA1, UA2, and UA3.

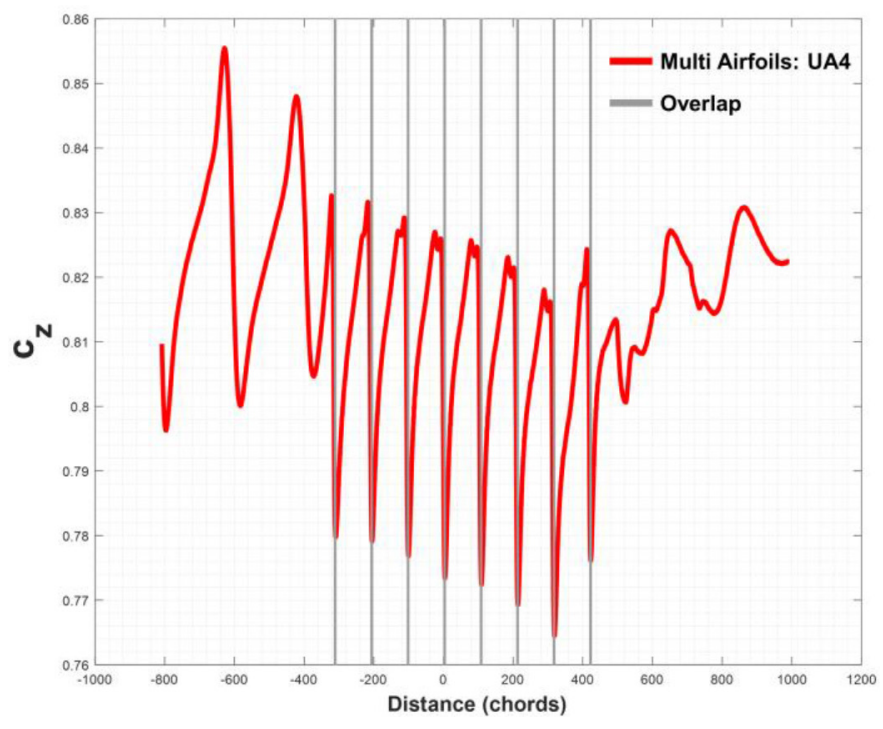

Figure 8. Multi airfoils crossing simulation of UA4 crossing lower airfoils 1-8 for $\mathrm{c}_{\mathrm{z}}$ versus distance to overlap ( $\mathrm{HC} 1: \mathrm{M}=0.47\left(\mathrm{~V}_{\text {tip }}=500 \mathrm{ft} / \mathrm{s}\right), \alpha=7^{\circ}, \mathrm{S} / \mathrm{c}=$ $6.21(\mathrm{~S}=2.33 \mathrm{ft}, \mathrm{c}=0.375 \mathrm{ft}))$, and $\mathrm{D} / \mathrm{c}=104.72(\mathrm{D}=39.27 \mathrm{ft}))$.

For the $\mathrm{HC} 1$ design, Fig. 9 a) and b) show the isolated airfoil, two airfoils crossing (UA), multi airfoils crossing (UA4) simulation results for $\mathrm{c}_{\mathrm{z}}$ and $\mathrm{c}_{\mathrm{x}}$ versus distance to overlap, respectively. Figure 9 c) and d) show the isolated airfoil, two airfoils crossing (LA), multi airfoils crossing (LA4) simulation results for $\mathrm{c}_{\mathrm{z}}$ and $\mathrm{c}_{\mathrm{x}}$ versus distance to overlap, respectively.

The upper and lower airfoil time histories show an overall decrease in $c_{z}$ for the multi airfoil (UA4 and LA4) simulation compared to the two-airfoil and isolated airfoil simulations. The opposite occurs for $c_{x}$, where the multi airfoil simulation see an increase in $c_{x}$ compared to the isolated and two airfoil simulations. 
For the two-airfoil simulation, the $\mathrm{c}_{\mathrm{z}}$ time history results show an increase before overlap and decrease after overlap and vice versa for $\mathrm{c}_{\mathrm{x}}$. The multiple airfoil results show an overall similar result, but with additional fluctuations. The additional fluctuations for the multiple airfoil cases is due to vortices interacting from other airfoils. Multiple vortices complicate the flow field and introduce time-varying loads on the airfoil.
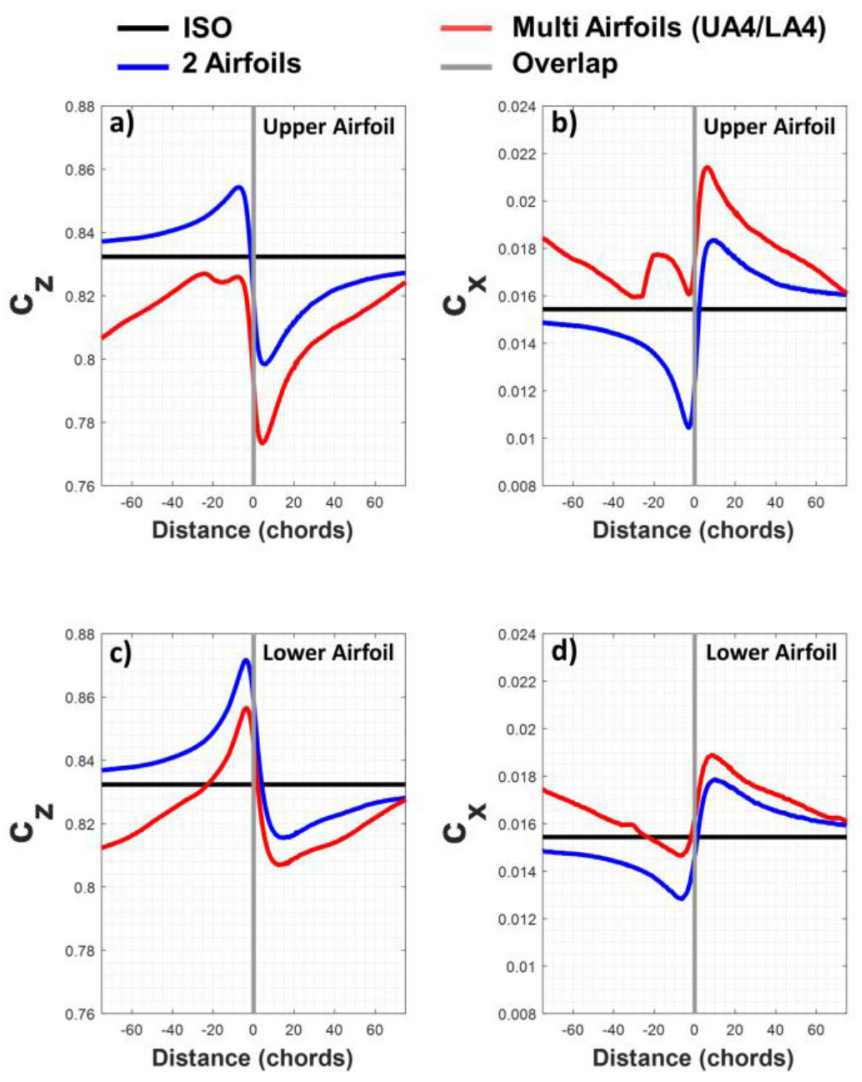

Figure 9. Isolated airfoil, two airfoils crossing (UA), multi airfoils crossing (UA4) for a) $c_{z}$ and b) $c_{x}$ versus distance to overlap, and an isolated airfoil, two airfoils crossing (LA), multi airfoils crossing (LA4) for c) $c_{z}$ and d) $c_{x}$ versus distance to overlap ( $\mathrm{HC} 1: \mathrm{M}=0.47\left(\mathrm{~V}_{\text {tip }}=500 \mathrm{ft} / \mathrm{s}\right), \alpha=7^{\circ}, \mathrm{S} / \mathrm{c}=6.21$ $(\mathrm{S}=2.33 \mathrm{ft}, \mathrm{c}=0.375 \mathrm{ft}))$, and $\mathrm{D} / \mathrm{c}=104.72(\mathrm{D}=39.27 \mathrm{ft}))$.

\section{Simulation of a Modern Coaxial Rotor Design}

A modern rotor design is simulated using eight NACA 0012 upper and lower airfoils at $\mathrm{M}=0.627\left(\mathrm{~V}_{\text {tip }}=700 \mathrm{ft} / \mathrm{s}\right), \alpha=5^{\circ}, \mathrm{S} / \mathrm{c}=4(\mathrm{~S}=2$ $\mathrm{ft}, \mathrm{c}=0.5 \mathrm{ft})$, and $\mathrm{D} / \mathrm{c}=83.8(\mathrm{D}=41.9 \mathrm{ft})$. Figure 10 shows $\mathrm{c}_{\mathrm{z}}$ versus distance to overlap for UA4 in the multi- airfoil simulation of the modern design. Similar to Fig. 8 , Fig. 10 shows three large fluctuations occur before the crossing event of UA4 with LA1, these three large fluctuations are due to UA4 traveling through the starting vortices of UA1 through UA3.

For the modern design, Fig. 11 a) and b) show the isolated airfoil, two airfoils crossing (UA), and multi airfoils crossing (UA4) simulation results for $\mathrm{c}_{\mathrm{z}}$ and $\mathrm{c}_{\mathrm{x}}$ versus distance to overlap, respectably. Figure 11 c) and d) show the isolated airfoil, two airfoils crossing (LA), multi airfoils crossing (LA4) simulation results for $\mathrm{c}_{\mathrm{z}}$ and $\mathrm{c}_{\mathrm{x}}$ versus distance to overlap, respectively.

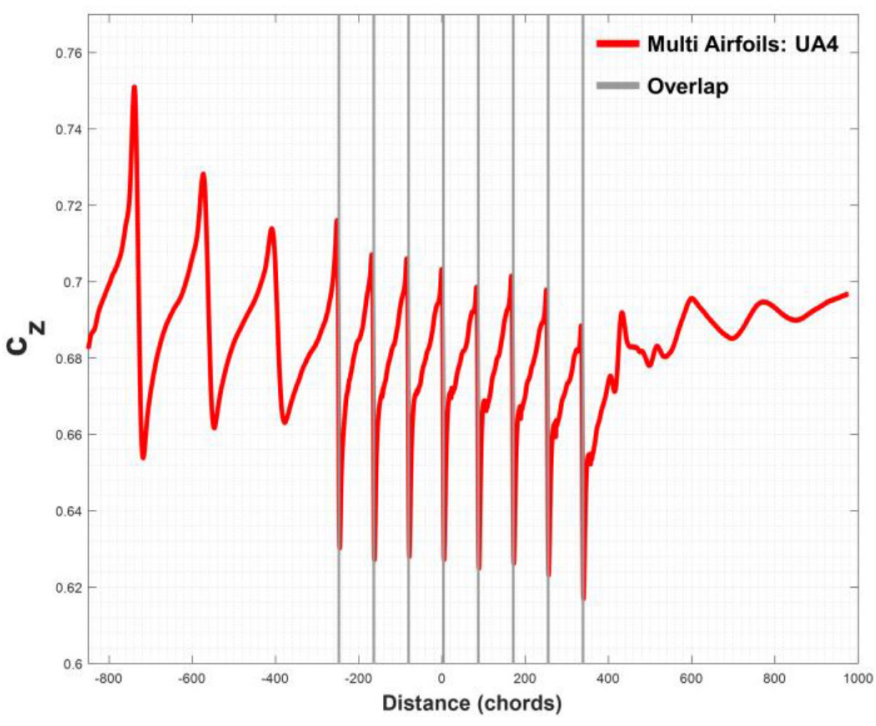

Figure 10. Multi airfoils crossing simulation of UA4 crossing lower airfoils 1-8 for $\mathrm{c}_{\mathrm{z}}$ versus distance to overlap (Modern: $\mathrm{M}=0.627\left(\mathrm{~V}_{\text {tip }}=700 \mathrm{ft} / \mathrm{s}\right), \alpha=$ $5^{\circ}, \mathrm{S} / \mathrm{c}=4(\mathrm{~S}=2 \mathrm{ft}, \mathrm{c}=0.5 \mathrm{ft})$, and $\mathrm{D} / \mathrm{c}=83.8(\mathrm{D}=41.9 \mathrm{ft})$ ).
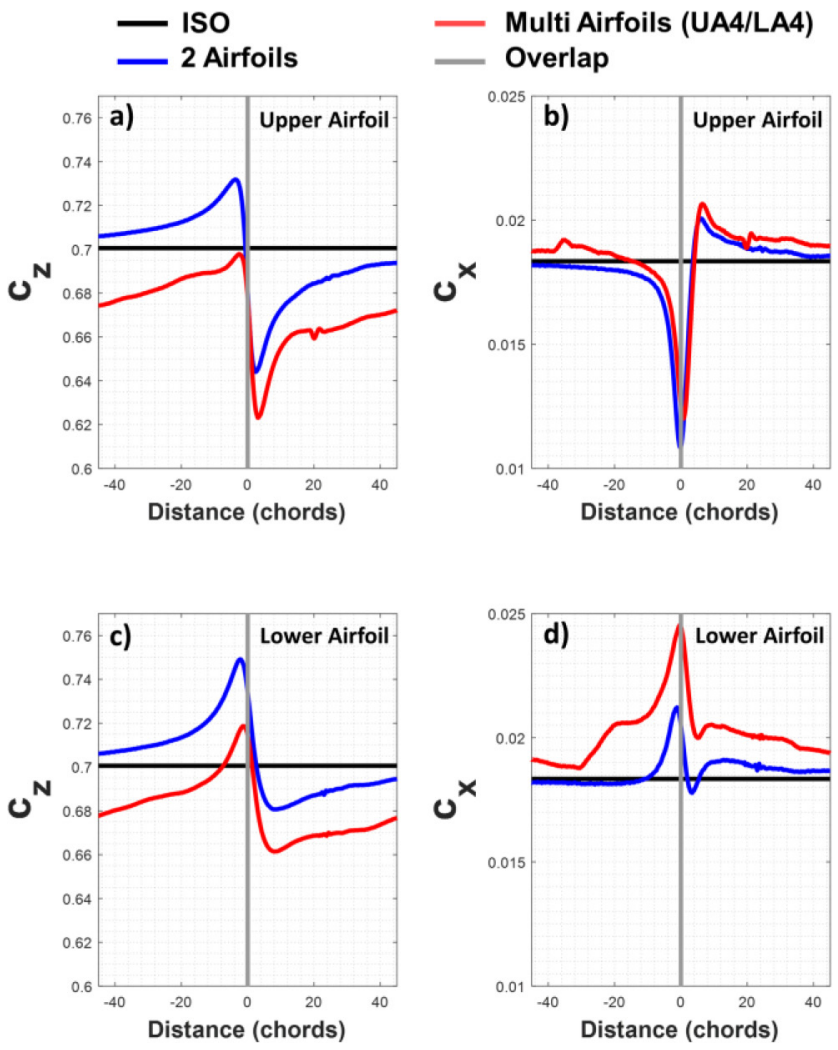

Figure 11. Isolated airfoil, two airfoils crossing (UA), multi airfoils crossing (UA4) for a) $c_{z}$ and b) $c_{x}$ versus distance to overlap, and an Isolated airfoil, two airfoils crossing (LA), multi airfoils crossing (LA4) for c) $c_{z}$ and d) $c_{x}$ versus distance to overlap (Modern: $\mathrm{M}=0.627\left(\mathrm{~V}_{\text {tip }}=700 \mathrm{ft} / \mathrm{s}\right), \alpha=5^{\circ}, \mathrm{S} / \mathrm{c}=4$ $(\mathrm{S}=2 \mathrm{ft}, \mathrm{c}=0.5 \mathrm{ft})$, and $\mathrm{D} / \mathrm{c}=83.8(\mathrm{D}=41.9 \mathrm{ft}))$.

The upper and lower airfoil time history showed an overall decrease in $\mathrm{c}_{\mathrm{z}}$ for the multi airfoil (UA4 and LA4) simulation compared to the two airfoil and isolated airfoil simulations. Comparing the upper airfoils (UA and UA4), the $\mathrm{c}_{\mathrm{x}}$ magnitude and minimum peak values 
are similar. The lower airfoils $\mathrm{c}_{\mathrm{x}}$ show a similar trend seen in Fig 9. d), where LA4 saw an overall increase in $\mathrm{c}_{\mathrm{x}}$ compared to LA and isolated airfoil simulations. Similar to the $\mathrm{HC} 1 \mathrm{c}_{\mathrm{z}}$ and $\mathrm{c}_{\mathrm{x}}$ results, the global trend is comparable (positive slope before overlap and negative slope after overlap), but the shed vorticity introduces additional disturbances.

Compared to the $\mathrm{HC} 1$ results, the modern design introduces effects of compressibility due to the increased Mach number $(\mathrm{M}=0.47$ to 0.627). In particular, the modern design results of $c_{x}$ versus distance for both UA4 and LA4, show a sharp peak at time of overlap, as seen by Barbely et al. [4] for high Mach numbers. This sharp peak could be the result of a weak shock formation.

\section{CONCLUDING REMARKS}

Previously, Barbely et al. [르, $\underline{3}$, and $\underline{4}]$ simulated two airfoils traveling in opposite directions using OVERFLOW CFD. The simulation retained effects of circulation, thickness, and compressibility for a coaxial rotor, but lacked information regarding the rotor wake/vortex sheet. A previous test condition of the Harrington rotor (HC1) was recalculated using grid adaption in OVERFLOW to better capture the airfoil wake and to further investigate the significance of shed vorticity. The isolated $\mathrm{HC} 1$ airfoil simulation flow field was subtracted from the two airfoil crossing simulation to isolate effects of shed vorticity due to two airfoils crossing. The vorticity contour was consistent with the computed shed vorticity $(-\mathrm{d} \Gamma / \mathrm{dt})$ for the upper airfoil. The vorticity contour plots revealed a large difference in vorticity compared to the isolated case. Because of this, a train of airfoils are simulated to further understand shed vorticity.

The current work simulates rotor wake/vortex effects in 2D by a train of airfoils separated by a horizontal distance (or phase). Eight airfoils on the upper rotor and eight airfoils on the lower rotor are simulated using OVERFLOW with the leading airfoils (UA1 and LA1) starting 200 chord lengths away from time of crossing (overlap at $1 / 4$ chord). For both the Harrington rotor 1 and the modern design simulation, the influence of the shed vorticity had a different aerodynamic effect compared to the two airfoil crossing simulation.

Results revealed the complex nature of the aerodynamic impulses generated by blade-blade interactions, with implications for aeroelastic loads and aeroacoustic sources. Simulating multiple airfoils gave the ability to understand the effect of shed vorticity for a coaxial rotor.

Specific findings are listed below:

The upper airfoil (UA4) for the multi airfoil simulation $c_{\mathrm{x}}$ results were similar in magnitude and minimum peak value compared to UA. The lower airfoil $c_{x}$ time history showed an overall increase in magnitude for the multi-airfoil simulation compared to the two airfoil simulation.
Comparing $\mathrm{c}_{\mathrm{z}}$ versus distance to overlap results for both design simulations, the two airfoil simulation results show a positive slope before overlap and a negative slope after. The multiple airfoil simulation revealed an overall similar result, but with a reduced mean value and additional fluctuations. The additional fluctuations in the $c_{z}$ and $c_{x}$ time history results for the multiple airfoil simulation cases are due to vortices interacting from other airfoils. Multiple vortices further complicate the flow field and introduce time-varying loads on the airfoil.

With an increased Mach number compared to the $\mathrm{HC} 1$ design, the modern design introduced additional effects of compressibility, particularly for the $c_{x}$ versus distance results. The overall shape of the $c_{x}$ curves indicates possible transonic effects in the flow field.

\section{REFERENCES}

1. Coleman, C. P., "A Survey of Theoretical and Experimental Coaxial Rotor Aerodynamic Research,” NASA TP- 3675, NASA, March 1997.

2. Barbely, N. L., Komerath, N. M., and Novak, L. A., "A Study of Coaxial Rotor Performance and Flow Field Characteristics," American Helicopter Society Aeromechanics Specialist's Conference, San Francisco, CA, January 20-22, 2016.

3. Barbely, N. and Komerath, N., "Coaxial Rotor Flow Phenomena in Forward Flight," SAE Technical Paper 2016-01-2009, 2016, doi: 10.4271/2016-01-2009.

4. Barbely, Natasha L., and Komerath, N.M., "Compressible 2D Flow Field Interaction of Two Contra-Rotating Blades." ASME 2016 International Mechanical Engineering Congress and Exposition, pp. V013T01A007V013T01A007. American Society of Mechanical Engineers, 2016.

5. Singh, Puneet, and Friedmann Peretz P.. "Application of Vortex Methods to Coaxial Rotor Wake and Load Calculations." 55th AIAA Aerospace Sciences Meeting. 2017.

6. Nichols, R. and Buning, P., User's Manual for OVERFLOW 2.2, NASA Langley Research Center, Hampton, VA, August 2010.

7. Harrington, R. D., "Full-scale-tunnel investigation of the static-thrust performance of a coaxial helicopter rotor," NACA TN-2318, NACA, March 1951.

8. Johnson, W., "Influence of Lift Offset on Rotorcraft Performance," NASA TP- 215404, NASA, November 2009.

\section{CONTACT INFORMATION}

\author{
Natasha Schatzman (formally Barbely) \\ NASA Ames Research Center \\ Mail Stop: 243R-11 \\ Moffett Field, CA 94035-0001 \\ Natasha.L.Barbely@nasa.gov \\ Office: (650) 604-5903
}

\section{ACKNOWLEDGMENTS}

The authors would like to thank the U. S. Army Aviation Development Directorate (ADD), with personal thanks to Dr. William Warmbrodt (NASA), Dr. Gloria Yamauchi (NASA), and Dr. Ben Sim (AFDD). 


\section{NOMENCLATURE}

c - chord (ft)

$\mathbf{c}_{\mathrm{x}}$ - coefficient of force in the $\mathrm{x}$ direction $\left(\frac{x_{\text {force }}}{\frac{1}{2} \rho V^{2} c}\right)$

$\mathbf{c}_{\mathrm{z}}$ - coefficient of force in the $\mathrm{z}$ direction $\left(\frac{z_{\text {force }}}{\frac{1}{2} \rho V^{2} c}\right)$

D - horizontal distance between airfoils (ft)

LAi - lower airfoil $(\mathrm{i}=1,2,3,4,5,6,7,8)$

$\mathbf{I}$ - lift per unit span (lbs.)

M - Mach number

$\mathbf{M}_{\text {tip }}$ - tip Mach number

$\mathbf{R}$ - rotor radius ( $\mathrm{ft}$ )

$\mathbf{t}$ - time $(\mathrm{s})$

UAi - upper airfoil $(\mathrm{i}=1,2,3,4,5,6,7,8)$

$\mathbf{V}_{\text {tip }}$ - blade tip speed (ft/s)

$\mathbf{S}$ - vertical distance between rotors or airfoils ( $\mathrm{ft}$ )

$\Gamma$ - circulation $\left(\mathrm{ft}^{2} / \mathrm{s}\right)$

This is a work of a Government and is not subject to copyright protection. Foreign copyrights may apply. The Government under which this paper was written assumes no liability or responsibility for the contents of this paper or the use of this paper, nor is it endorsing any manufacturers, products, or services cited herein and any trade name that may appear in the paper has been included only because it is essential to the contents of the paper.

Positions and opinions advanced in this paper are those of the author(s) and not necessarily those of SAE International. The author is solely responsible for the content of the paper 